https://helda.helsinki.fi

\title{
Time Markers and Temporal Illusions
}

\section{Arstila, Valtteri}

Palgrave Macmillan

2019

Arstila , V 2019 , Time Markers and Temporal Illusions . in V Arstila , A Bardon, S Power \& A Vatakis (eds), The Illusions of Time. : Philosophical and Psychological Essays on Timing and Time Perception. . vol. 2019 , Palgrave Macmillan, Cham, Sveitsi , pp. 339-357 .

http://hdl.handle.net/10138/334616

unspecified

acceptedVersion

Downloaded from Helda, University of Helsinki institutional repository.

This is an electronic reprint of the original article.

This reprint may differ from the original in pagination and typographic detail.

Please cite the original version. 
Time markers and temporal illusions

\title{
Valtteri Arstila
}

\author{
Turku Institute for Advanced Studies \& Department of Philosophy \\ University of Turku, Finland
}

\begin{abstract}
According to the thesis of temporal isomorphism, the experienced order of events in the world and the order in which experiences are processed in the brain are the same. The thesis is encompassed in the brain-time view, a popular view on the literature of the temporal illusions. The view is commonly contrasted with the event-time view, which maintains that the experienced order of events reflects the order in which the events occur in the world. This chapter focuses on the conflict between the two views in the contexts of perceptual asymmetry in visual perception and temporal order judgment tasks. It is argued that both views mean slightly different things in these contexts. Accordingly, it is possible for one to endorse both the brain-time view and the event-time view at the same time. On the broader perspective, the chapter illustrates how time order is employed differently by various perceptual processes, resulting in different characteristics from implicit and explicit time perception.
\end{abstract}




\section{Introduction}

Three notions of time are commonly distinguished in the literature of the temporal illusions. The event time is the time when an external event occurs. The brain time of experience (brain time for short) is the time when the neural processes that realize the experience of the event occur (i.e., neural correlates of experiences). The subjective time of experience (subjective time for short) refers to the time in which an event is experienced to occur. Much of the discussion on the temporal illusions in recent years has been about the relationship between the last notion of time and the other two.

The position known as the brain-time view maintains that the distinction between subjective time and brain time is merely conceptual. In reality, subjective time and brain time are isomorphic. The brain-time view is contrasted with the event-time view, according to which subjective time mirrors (or aims to mirror) event time rather than brain time. It is important to notice, however, that both views have been understood in two different views. As a result, one version of the event-time view is compatible with the brain-time view's claim that subjective time and brain time are isomorphic. For this reason, I will refer to this claim as the thesis of temporal isomorphism rather than a brain-time view.

This chapter aims to explicate the ambiguity in the two views and how they relate to the thesis of temporal isomorphism. The aim is not to argue for the truth of the thesis of temporal isomorphism nor for a specific notion of the brain-time view or the event-time view. ${ }^{\mathrm{i}}$ The related philosophical literature is not so relevant to this volume because it mostly concerns the theories of the temporal structure of consciousness, rather than temporal illusions. ${ }^{\text {ii }}$ Moreover, the focus of this chapter is on temporal order illusions, although the views and the thesis apply to duration illusions too. In the discussed context, the thesis states that the order 
of events represented in the experiences (i.e., subjective time order) is the same as the temporal order of neural processing underlying the experiences (i.e., brain time order).

In what follows, I begin by summarizing the parts of Daniel Dennett and Marcel Kinsbourne's Time and the Observer (1992) that directly relate to the thesis. It is the most discussed exposition of the thesis of temporal isomorphism; the arguments in the paper are frequently cited as reasons to reject the thesis. Moreover, the two versions of the event-time view concur with two alternatives Dennett and Kinsbourne present for the thesis of temporal isomorphism. The succeeding sections cover these two versions of the event-time view along with the ambiguity of the brain-time view. The first version concerns the temporal order judgments of changes within one stimulus; the second version concerns temporal order judgments between the occurrence of two stimuli. The final section summarizes the discussion and situates it in the context of whether or not experience occurs in one mental timeline.

\section{The thesis of temporal isomorphism and alternative views}

In Time and the Observer, Dennett and Kinsbourne contrasted the Cartesian theater model of consciousness with the Multiple Drafts model of consciousness. Both models hold that feature-detections and discriminations of the features of stimuli are done at different parts of the cortex. That is, the processing of the features is localized. According to the Cartesian theater model, we become conscious of the localized discriminations when they are represented at the hypothetical Cartesian theater. This is a place "where it all comes together,' and the discriminations in all modalities are somehow put into registration and 'presented' for subjective judgment." (Dennett \& Kinsbourne 1992, 183) The subjective time of the experiences is determined by the time the localized discriminations are (re)presented at the Cartesian theater. This means that the subjective time order of experienced events is the 
same as the order in which experiences of the events occur. In short, the Cartesian theater model entails the thesis of temporal isomorphism.

Dennett and Kinsbourne's objection to the Cartesian theater model makes use of three temporal anomalies - the backward metacontrast masking effect, the color phi phenomenon, and the cutaneous rabbit illusion-each of which brings about incorrect reports of the stimuli. ${ }^{\text {iii }}$ As an example, consider the ordinary phi phenomenon. Here, subjects are presented with spatially separated static stimuli (flashes) with a short temporal gap between them, and they report seeing that something moves between the location of the presented stimuli. The temporally anomalous part of the illusion is that the apparent motion can be processed in the brain only after the second stimulus is processed — or so Dennett and Kinsbourne arguebecause otherwise the motion would not have direction. Thus the order of neural processing (first stimulus, second stimulus, apparent motion) does not appear to match with the reported order of experiences (first stimulus, apparent motion, second stimulus).

The described discrepancy raises the question of whether our report is correct or not. If the Stalinesque revision took place, then the report is correct. We see motion before the second stimulus, because the order of localized discriminations is tampered with before they reach the Cartesian theater. In this case, the order in which localized discriminations are processed is different from the order in which they arrive at the Theater. If the Orwellian revision takes place, then our experience of the second stimulus precedes our experience of motion, but we report them in the incorrect order because our memory of the order of events is tampered with. In the two possible scenarios, only the Stalinesque revision concurs with the thesis of temporal isomorphism.

According to Dennett and Kinsbourne, the Cartesian theater model necessitates that there is a way to distinguish which scenario is correct. Their main claim in the paper is that there is no 
such way. Consequently, they maintain that the Cartesian theater model is incorrect, and, with it, the thesis of temporal isomorphism is questionable as well. Dennett and Kinsbourne argue further that there is no reason that an experience of stimulus $S_{1}$ preceding stimulus $S_{2}$ needs to be accomplished by, first, a neural state realizing an experience of $S_{1}$ and then a neural state realizing an experience of $S_{2}$. The temporal order is not determined by the order in which the localized discriminations are represented at the Cartesian theater; it is determined by the temporal content of the individual representings of the events.

Dennett and Kinsbourne present two possibilities for what it means, in practice, to separate the time in the content of experience (experienced time) and the time of the vehicle of experience (the neural events realizing the experience). It is worth noting, however, that they do not explicate either possibility in detail. Instead, they provide analogies meant to illustrate the ideas behind them.

According to the first possibility, which I refer as the time-stamp view, the temporal properties of experience utilize time-stamps, a kind of abstract temporal label, linked to the experienced contents. Time-stamps have temporal information as their content. Dennett and Kinsbourne's analogy for timestamps is postmarks on letters: the postmarks represent the order in which letters are sent, not the time when are received. What matters for the subjective order of experiences are the postulated time-stamps that accompany the experiences, not the order in which localized discriminations are represented at the hypothetical Cartesian theater.

Dennett and Kinsbourne call the second possibility the content-sensitive settling. Their analogy for this possibility comes from matching the auditory and visual tracks of a film. Such matching can be done by determining abrupt changes in both tracks and trying to find 
the best match between the changes, even when we do not know much about the film or even understand the spoken language.

Dennett and Kinsbourne $(1992,189)$ prefer the content-sensitive settling view over the timestamp view because they regard the first "a cheaper, less foolproof but biologically more plausible." Nonetheless, it is the latter position that is commonly attributed to them in discussions of temporal illusions. This is possibly because part of their argument requires the timestamp view. For example, the content-sensitive settling cannot explain the experiences of features that are not in the stimuli, like an experience of motion in the phi phenomenon, because it only adjusts the relative order of experienced events. Moreover, the contentsensitive settling does not specify the stage of processing in which the settling occurs. For instance, it could take place before localized discriminations reach the Cartesian Theater or even before localized discriminations. These possibilities mean that the content-sensitive settling is compatible with the Cartesian theater model and the thesis of temporal isomorphism. ${ }^{\text {iv }}$

To summarize the relevant points briefly, the Cartesian theater model of consciousness maintains that we are conscious of localized discriminations of stimulus features only when they are represented in the Cartesian theater. Concurring with the thesis of temporal isomorphism, the subjective time order mirrors the brain time order, where brain time is the order of localized discriminations represented in the Cartesian theater. Since localized discriminations, formed in the various regions of the cortex, remain unconscious if they are not represented in the Cartesian theater, conscious and unconscious perception can have different temporal properties and structures. Finally, after rejecting the Cartesian theater and the thesis of temporal isomorphism, Dennett and Kinsbourne propose that the subjective time of experiences is determined by the temporal content of localized discriminations. They 
presented two ways in which this could happen: the time-stamp view and the content sensitive settling.

\section{Perceptual asynchrony in visual perception}

One research topic in which the brain-time and the event-time views have figured in prominently is the alleged perceptual asynchrony of changes of visual features. The central finding in question is that when a moving stimulus changes its color and direction of movement simultaneously and repeatedly, observers report that the change in color occurs 60-100 milliseconds before the change in direction. This effect takes a little while to occur as it is not reported in the first changes of the features or if the features change only once (Nishida \& Johnston 2002).

The brain-time view, sometimes also called the brain-time account and the brain-time theory of perception (Arnold 2005, 2010), explains the findings by maintaining that colors and changes in color are processed faster than motion and motion reversals (Moutoussis \& Zeki 1997, 2002). As Zeki and Bartels $(1999,234)$ put it, their "result implies that the color processing system reaches its perceptual endpoint before the motion processing system." The difference in perceptual latency is reflected correctly in the judgments of the temporal features of the events. One implication of this difference is that the difference between the cases when the changes are asynchronous and those in which they are not (e.g., the stimuli are not shown repeatedly) is because of some sort of adaptation to the asynchronous changes. It is not because our reports change from being incorrect to being correct. Since the subjective order of the changes is the same as the order in which the experiences of changes are neurally processed, the brain-time view subscribes to the thesis of temporal isomorphism. 
The brain-time view is attributed to Semir Zeki and his colleagues, who were first to report the described findings. Zeki took the findings, in concurrence with the known functional properties of the visual cortex, to support his theory of consciousness (Zeki 2007; Zeki \& Bartels 1999). This is significant because the subsequent discussion of perceptual asynchrony in vision and the brain-time view has been understood in terms of Zeki's theory of consciousness, which differs from the Cartesian theater model of consciousness.

For the topic at hand, the most important difference between these models of consciousness is that, in Zeki's theory, the activity of the parts of the visual cortex that process a visual feature can be a neural correlate of conscious experience. In Dennett and Kinsbourne's terms, Zeki argues that, when localized discrimination is finished, we can become conscious of the detected visual feature without it being represented in the Cartesian theater. Thus, there is no separation between the time when the processing of localized discrimination is finished and the time when we experience the result.

Citing Dennett and Kinsbourne, Johnston and Nishida (2001, R428) argue that the brain-time view faces "some thorny philosophical problems," at the root of which is the commitment to the thesis of temporal isomorphism. Moreover, they maintain that "a brain time mechanism is poorly designed in the sense that processing delay is added to event time estimation." (Nishida \& Johnston 2010, 286) And indeed, if the subjective time is inherently linked to the brain time, then the subjective timing of events is influenced by the neural latencies. This makes deducing the event time, the actual time of external events, practically impossible. After all, to do so, we would need to take into account the neural latencies, which differ both within one sense modality (e.g., Allik \& Kreegipuu 1998; Maunsell et al. 1999) and between sense modalities, and yet neurons are spatiotemporally limited in the sense that they do not have the information about the various latencies at their disposal (Scharnowski, Rees, \& Walsh 2013). ${ }^{\mathrm{v}}$ 
Nishida and Johnston's $(2002,2010)$ method of avoiding this problem is based on two ideas. First, they distinguish between two types of temporal changes. Determining the first-order temporal changes, called transitions, requires that the stimulus is compared in two points in time. For instance, we can detect the change in color if we compare the color of a stimulus at times $t_{1}$ and $t_{2}$. Determining the second-order temporal changes, called turning points, requires that the stimulus is compared in three points in time. For instance, they argue, the detection of motion reversal demands that we compare the spatial position of the stimulus at times $t_{1}, t_{2}$, and $t_{3}$. Consequently, the implication is that even if a transition and a turning point were to co-occur at $t_{2}$, determining the time of turning points takes longer than determining the time of transitions. Nishida and Johnston's results as regards synchrony between different types of transitions and turning points provide support for this claim. Given that the change in color and in the direction of motion are different types of temporal changes, simultaneous changes in them would not be judged to be simultaneous.

In effect, Nishida and Johnston's $(2002,365)$ claim means that the reports of perceptual asynchrony are "a result of an error of subjective temporal judgments" rather than asynchrony in the experiences of changes in color and motion. As such, it is compatible with the thesis of temporal isomorphism; the thesis concerns only the relationship between subjective time and brain time. It is also compatible with Dennett and Kinsbourne's view that subjective judgments occur when localized discriminations have been presented at the Cartesian theater. That is, their position allows that our temporal judgments of experiences can be incorrect.

The second idea that Nishida and Johnston put forward is that temporal judgments are based on time-markers, markers that are themselves based on early neural activity. The resulting view, which they call the time-marker view, distances them from the way in which the relationship between brain time and subjective time was envisaged in the Cartesian theater 
model. By time-markers (also temporal markers), they mean "temporally localized representations of salient temporal features" $(2002,360)$, which are based on "the temporal pattern of the neural activity elicited by [external] events" $(2002,366)$. The claim that the relevant neural activity takes place very early on in the processing hierarchy has the consequence that the processing of time-markers is dissociated from the finished localized discriminations and conscious experiences, and especially the timing thereof. Since temporal order judgments utilize time-markers rather than the brain time of experience, their view resembles Dennett and Kinsbourne's time-stamp view in this respect.

Moreover, the idea allows Nishida and Johnston mostly to avoid the problem they thought the brain-time view suffered from. If the subjective time is linked to the time-markers grounded on the early neural activation caused by the external events, then the subjective time reflects the time when the processing of a feature is commenced rather than finished. In this case, the differences in neural latencies and the processing times in later processing stages do not influence the temporal judgments. This, in turn, means that subjective time and temporal judgments mirror the event time rather than the brain time. In short, Nishida and Johnston endorse the event-time view.

Nishida and Johnston's notion of a time-marker also resembles Libet's notion of a cortical time marker. Briefly, Libet argued that conscious experience of a tactile stimulus requires cortical processing for 500 milliseconds and that subjectively the stimulus is felt to occur already around the time when the processing is commenced. That is, the subjective time of experience and the brain time of experience come apart-the subjective time of skin sensations are "referred back" to the time when the processing commenced rather than when the neural processing of the stimulus becomes "adequate" to bring about the sensations. Moreover, Libet hypothesized that the first components of the evoked potential caused by the direct stimulation of the skin are used as cortical time-markers based on which temporal 
judgments are made, and that determines the subjective time of experience. Since the first evoked potential in the cortex occurs very fast after the presentation of the stimulus (in some of Libet's experiments, 15 milliseconds after the stimulation), the neural activity used for temporal coding mirrors the timing of external events certainly more closely than if all neural latencies would be taken into account.

\section{The views and temporal order judgments}

The second topic in which notions of the brain-time view and the event-time view figure is explanations of the temporal order perception of separate stimuli. The relevant phenomena requiring explanation are when two simultaneous stimuli are perceived as non-simultaneous, and when two non-simultaneous stimuli are perceived as simultaneous. For example, a dim flash shown at the same time as a bright flash is perceived as occurring after the flash. Or, when a toe is touched tens of milliseconds before a nose, the two are perceived as occurring at the same time. Similar illusions also happen with multimodal stimuli; for example, a flash and a sound can appear simultaneous even though they are separated by a hundred milliseconds.

One straightforward explanation for these results appeals to latency differences: the simultaneous stimuli are perceived as non-simultaneous because the neural processing of them took different amounts of time. In accordance with this explanation, the empirical evidence suggests that a bright stimulus is processed faster than a dimmer stimulus, and it takes longer time for a signal from a toe to reach the cortex than from a nose. ${ }^{\mathrm{vi}}$ In this context, this explanation has been called the brain-time view. ${ }^{\text {vii }}$ Again, this explanation concurs with the thesis of temporal isomorphism. 
The brain-time view has been taken to be challenged by evidence that we adapt to nonsimultaneous multimodal stimuli. One interpretation of this evidence is that the adaptation is achieved by the brain physically aligning the timing of the signals it receives (e.g., Harrar \& Harris 2008; Harris, Harrar, Jaekl, \& Kopinska 2010; Navarra, García-Morera, \& Spence 2012). The suggested adaptation is what Dennett and Kinsbourne's content-sensitive settling does. If the brain recalibrates the sensory signals in this manner, then the explanation of temporal order perception is no longer a matter of simple latency differences. Instead, the possibly adaptive nature of temporal order perception increases the accuracy of the subjective time in relation to the actual order of events. Since, in this case, the subjective time reflects the event time - thus resembling the event-time view of the previous section-this position has also been called the event-time view.

Two things are worth noting here. First, the adaptation to a non-simultaneous multimodal stimulus can also be explained by shifts in decision criteria (Yarrow, Jahn, Durant, \& Arnold 2011). Unlike the previous interpretation of the results, the adaptation happens at the postexperiential decision level. Thus, the explanation concurs with the brain-time view. Second, in this context, the crucial difference between the subjective time view and the event-time view concerns the possible existence of an adaptive mechanism that temporally aligns the sensory signals. This crucial difference means that, departing from how the event time is implemented in the time-marker view, the event-time view is in theory compatible with the thesis of temporal isomorphism. The two are also likely to be compatible in practice, because the adaptation has been postulated to take place in early sensory pathways (e.g., Heron, Roach, Hanson, McGraw, \& Whitaker 2012; Maravita, Bolognini, Bricolo, Marzi, \& Savazzi 2008). If correct, then the subjective time of experience and the neural time of experience could still be isomorphic. 


\section{Further considerations}

This chapter focused on views about the relationships between subjective time, brain time, and event time. The brain-time view(s) subscribe to the thesis of temporal isomorphism-an event is perceived to occur at the time neural signals the event evokes reach the processes responsible for consciousness. However, it was argued that there are two versions of the view, and they differ in two respects.

First, the two versions differ regarding the processes responsible for consciousness. This disagreement is fundamentally over the neural correlates of consciousness that supposedly match the subjective time of experience. In the first version, the perceptual endpoint is reached when the process that determines features of the perceived event finishes. The second version does not comment on the perceptual endpoint. Hence, it is open to the more commonly held view that conscious states are somehow unified. Unlike Zeki's theory, the processes that determine the features of the perceived event are separate and precede the processes responsible for consciousness.

Second, the versions differ on their position towards the processes preceding consciousness. The first version is compatible with there being unconscious processes that aim to increase the match between subjective time and event time; the brain-time view as understood in the temporal order judgment literature rules out such unconscious processes.

The crux of the event-time view(s) is the idea that subjective time tracks or aims to track event time. In practice, this idea has been implemented in two very different ways. In Nishida and Johnston's time-marker view, which can be thought as inspired by Dennett and Zeki's time-stamp view, subjective time utilizes time-markers. The processing of time-markers happens early in the processing hierarchy and is largely dissociated from the processing of the content of experiences (localized discriminations). In the context of typical temporal 
order judgment literature, in turn, event time refers to a view in which subjective time aims to track event time by temporally aligning the propagating sensory signals. In practice, this is what Dennett and Zeki called content-sensitive settling. Because such adaptation is thought to happen before experiences are formed, unlike the time-marker view, this view is compatible with the thesis of temporal isomorphism. An interesting consequence of this compatibility is that the event-time view as understood in typical temporal order judgments counts as a braintime view in the perceptual asynchrony literature. This is because the literature is only concerned about the time when the perceptual end is reached and allows some adaptation to the asynchronous stimuli to occur.

Let me end by briefly commenting on a recent claim by Alex Holcombe, that experience does not comprise a single ordered timeline (Holcombe 2015). He maintains that meaningfully grouping and integrating stimuli is more important than ordering events in a timeline. On my view, the truth of the claim depends on the perspective one takes on the timeline.

If one approaches the issue of the timeline from the perspective of perceptual mechanisms, then it is most certainly correct. As mentioned several times in this paper, feature-detections and discriminations of the features of stimuli are done at different parts of the cortex. Although many of these processes utilize temporal information, which differs from one process to another, they are not usually involved in the processing of temporal features. Accordingly, they might preserve temporal information, but it is done by transforming the information to the non-temporal content of experiences. In practice, this means that the temporal information can be implicitly present in our experiences.

In some occasions, we can even use that information to make temporal judgments in which the temporal resolution is a magnitude smaller than the typical temporal order threshold (2-5 milliseconds versus 30-40 milliseconds). For example, visual stimuli can bring about 
experiences of apparent motion. In these cases, the direction of motion can be used to determine which stimulus was presented first (Sweet 1953; Wehrhahn \& Rapf 1992; Westheimer \& McKee 1977). In the auditory modality, loudness, pitch, and other micropatterns can be used to determine the order of stimuli (Babkoff \& Sutton 1963; Efron 1973; Fostick \& Babkoff 2013). For example, when two auditory stimuli are presented a few milliseconds apart, the first stimulus sounds louder than the second stimulus. Interestingly, at least in the cases described, the low temporal threshold is because the stimuli are presented so close in time that there is a mutual influence between their respective processes. When the stimulus onset asynchrony is larger, the cross-stimuli influence disappears. For example, subjects are better at determining the temporal order of two auditory stimuli when they are separated by less than four milliseconds than when they are separated by ten milliseconds (Babkoff 1975).

Holcombe's claim also receives support from the findings that grouping and binding do not require subjective synchrony of the grouped and bound features (Fahle \& Koch 1995; Guttman, Gilroy, \& Blake 2007). In fact, studies on audiovisual perception even suggest that subjective simultaneity is not optimal for subjective integration of the stimuli. The integration of two stimuli is better when they deviate from the point of subjective simultaneity determined in the temporal order tasks (Freeman et al. 2013; Ipser, Karlinski, \& Freeman 2018).

One interpretation for these results is that there is no ordered single timeline. Then again, it is not clear that the debate between the brain-time view and the event-time view even pertains to the sort of timeline Holcombe discusses. After all, at least what the brain-time view and the latter version of the event-time view maintain is that experiences are temporally ordered by the order in which they reach their perceptual endpoint. 
The timeline in question differs from the one implied by Holcombe. While it still consists of implicit timing, the nature of the implicitness is different. The temporal order of events is still only implicitly represented in the brain-time view and the latter version of the event-time view as well. However, in these cases, the order is represented by the order of experiences and not by, say, the direction of motion. Nonetheless, paraphrasing William James's remark - agreed by most philosophers and motivating the lively debate concerning the temporal structure of consciousness - the succession of experience is not the experience of succession. The latter needs to be separately represented. This claim concurs with Dennett and Kinsbourne's definition of the Cartesian theater, according to which it is the place where discriminations were “"presented' for subjective judgment." That is, the order of experiences is not the same as the judged order of experiences.

Moreover, neither of the views make claims about the mechanisms for reporting temporal properties. Thus, again, these mechanisms can utilize different information or have different decision criteria as suggested by simultaneity judgments, temporal order judgments, and reaction time studies (see, e.g., Jaśkowski 2014; Steglich \& Neumann 2000; Sternberg \& Knoll 1973; Tappe, Niepel, \& Neumann 1994). This too supports the idea that, considered from the perspective of perceptual mechanisms, Holcombe's claim is sound. However, his claim does not pertain to the question of whether there is a single ordered timeline of experiences per se. The plausibility of a single ordered timeline is crucially dependent on what we mean by such an idea. 


\section{References}

Allik, J., \& Kreegipuu, K. (1998). Multiple Visual Latency. Psychological Science, 9(2), 135-138. http://doi.org/10.1111/1467-9280.00025

Arnold, D. H. (2005). Perceptual pairing of colour and motion. Vision Research, 45(24), 3015-3026. http://doi.org/10.1016/j.visres.2005.06.031

Arnold, D. H. (2010). Relative timing and perceptual asynchrony. In R. Nijhawan \& B. Khurana (Eds.), Space and time in perception and action (pp. 254-277). Cambridge, UK: Cambridge University Press.

Arnold, D. H., Ong, Y., \& Roseboom, W. (2009). Simple differential latencies modulate, but do not cause the flash-lag effect. Journal of Vision, 9, 1-8. http://doi.org/10.1167/9.5.4.

Arnold, D. H., \& Wilcock, P. (2007). Cortical processing and perceived timing. Proceedings of the Royal Society B: Biological Sciences, 274(1623), 2331-6.

Arstila, V. (2015). Keeping postdiction simple. Consciousness and Cognition, 38, 205-216. http://doi.org/dx.doi.org/10.1016/j.concog.2015.10.001

Babkoff, H. (1975). Dichotic temporal interactions: Fusion and temporal order. Perception \& Psychophysics, 18(4), 267-272. http://doi.org/10.3758/BF03199373

Babkoff, H., \& Sutton, S. (1963). Perception of Temporal Order and Loudness Judgments for Dichotic Clicks. The Journal of the Acoustical Society of America, 35(4), 574-577. http://doi.org/10.1121/1.1918537

Chappell, M., \& Hine, T. (2004). Events before the flash Do influence the flash-lag magnitude. Vision Research, 44(3), 235-239. http://doi.org/10.1016/j.visres.2003.09.021

Dennett, D. C., \& Kinsbourne, M. (1992). Time and the observer. Behavioral and Brain 
Sciences, 15(2), 183-247.

Efron, R. (1973). Conservation of temporal information by perceptual systems. Perception \& Psychophysics, 14(3), 518-530. http://doi.org/10.3758/BF03211193

Fahle, M., \& Koch, C. (1995). Spatial displacement, but not temporal asynchrony, destroys figural binding. Vision Research, 35(4), 491-494. http://doi.org/10.1016/00426989(94)00126-7

Fostick, L., \& Babkoff, H. (2013). Different Response Patterns Between Auditory Spectral and Spatial Temporal Order Judgment (TOJ). Experimental Psychology, 60(6), 432443. http://doi.org/10.1027/1618-3169/a000216

Freeman, E. D., Ipser, A., Palmbaha, A., Paunoiu, D., Brown, P., Lambert, C., ... Driver, J. (2013). Sight and sound out of synch: Fragmentation and renormalisation of audiovisual integration and subjective timing. Cortex, 49(10), 2875-2887. http://doi.org/10.1016/j.cortex.2013.03.006

Grush, R. (2007). Time and experience. In T. Müller (Ed.), Philosophie der Zeit (pp. 1-18). Frankfurt: Klosterman.

Grush, R. (2009). The temporal content of perceptual experience. In J. Symons \& P. Calvo (Eds.), The Routledge Companion to Philosophy of Psychology. Abingdon: Routledge.

Guttman, S. E., Gilroy, L. A., \& Blake, R. (2007). Spatial grouping in human vision: temporal structure trumps temporal synchrony. Vision Research, 47(2), 219-30. http://doi.org/10.1016/j.visres.2006.09.012

Harrar, V., \& Harris, L. R. (2008). The effect of exposure to asynchronous audio, visual, and tactile stimulus combinations on the perception of simultaneity. Experimental Brain Research, 186(4), 517-24. http://doi.org/10.1007/s00221-007-1253-0 
Harris, L. R., Harrar, V., Jaekl, P., \& Kopinska, A. (2010). Mechanisms of simultaneity constancy. In R. Nijhawan \& B. Khurana (Eds.), Space and time in perception and action (pp. 232-253). Cambridge: Cambridge University Press.

Heron, J., Roach, N. W., Hanson, J. V. M., McGraw, P. V., \& Whitaker, D. (2012). Audiovisual time perception is spatially specific. Experimental Brain Research, 218(3), 477-485. http://doi.org/10.1007/s00221-012-3038-3

Holcombe, A. (2015). The Temporal Organization of Perception. (J. Wagemans, Ed.)The Oxford Handbook of Perceptual Organization. Oxford: Oxford University Press. http://doi.org/10.1093/oxfordhb/9780199686858.013.057

Ipser, A., Karlinski, M., \& Freeman, E. D. (2018). Correlation of Individual Differences in Audiovisual Asynchrony Across Stimuli and Tasks: New Constraints on Temporal Renormalization Theory. Journal of Experimental Psychology: Human Perception and Performance, 44(8), 1283-1293. http://doi.org/10.1037/xhp0000535

Jaśkowski, P. (2014). What Determines Simultaneity and Order Perception? In V. Arstila \& D. Lloyd (Eds.), Subjective Time: The Philosophy, Psychology, and Neuroscience of Temporality (pp. 379-408). Cambridge, MA: MIT Press.

Johnston, A., \& Nishida, S. (2001). Time perception: brain time or event time? Current Biology, 11(11), R427-R430.

Kelly, S. D. (2005). The puzzle of temporal experience. In A. Brook \& K. Akins (Eds.), The philosophy and neuroscience (pp. 1-26).

Kiverstein, J., \& Arstila, V. (2013). Time in Mind. In A. Bardon \& H. Dyke (Eds.), Blackwell companion to the philosophy of time (pp. 444-469). Oxford: Wiley-Blackwell.

Köhler, W. (1947). Gestalt Psychology: An Introduction to New Concepts in Modern 
Psychology. New York: Liveright.

Kopinska, A., \& Harris, L. R. (2004). Simultaneity constancy. Perception, 33(9), 1049-1060.

Lee, G. (2014). Temporal Experience and the Temporal Structure of Experience. Philosopher's Imprint, 14(3), 1-21.

Maravita, A., Bolognini, N., Bricolo, E., Marzi, C. A., \& Savazzi, S. (2008). Is audiovisual integration subserved by the superior colliculus in humans? NeuroReport, 19(3), 271275. http://doi.org/10.1097/WNR.0b013e3282f4f04e

Maunsell, J. H. R., Ghose, G. M., Assad, J. A., Mcadams, C. J., Boudreau, C. E., \& Noerager, B. D. (1999). Visual response latencies of magnocellular and parvocellular LGN neurons in macaque monkeys. Visual Neuroscience, 16(1), 1-14. http://doi.org/10.1017/S0952523899156177

Mölder, B. (2014). Constructing Time: Dennett and Grush on Temporal Representation. In Subjective Time: The Philosophy, Psychology, and Neuroscience of Temporality.

Moutoussis, K., \& Zeki, S. (1997). A direct demonstration of perceptual asynchrony in vision. Proceedings of the Royal Society B: Biological Sciences, 264(1380), 393-9. http://doi.org/10.1098/rspb.1997.0056

Moutoussis, K., \& Zeki, S. (2002). The relationship between cortical activation and perception investigated with invisible stimuli. Proceedings of the National Academy of Sciences, 99(14), 9527-9532.

Navarra, J., García-Morera, J., \& Spence, C. (2012). Temporal adaptation to audiovisual asynchrony generalizes across different sound frequencies. Frontiers in Psychology, 3. http://doi.org/10.3389/fpsyg.2012.00152

Nishida, S., \& Johnston, A. (2002). Marker correspondence, not processing latency, 
determines temporal binding of visual attributes. Current Biology, 12, 359-368.

Nishida, S., \& Johnston, A. (2010). The time marker account of cross-channel temporal judgments. In R. Nijhawan \& B. Khurana (Eds.), Space and time in perception and action (pp. 278-300). Cambridge, UK: Cambridge Univ Press.

Phillips, I. (2014). The Temporal Structure of Experience. In V. Arstila \& D. Lloyd (Eds.), Subjective Time: The Philosophy, Psychology, and Neuroscience of Temporality (pp. 139-158). Cambridge, MA: MIT press.

Reutimann, J., Yakovlev, V., Fusi, S., \& Senn, W. (2004). Climbing neuronal activity as an event-based cortical representation of time. The Journal of Neuroscience, 24, 32953303.

Scharnowski, F., Rees, G., \& Walsh, V. (2013). Time and the brain: Neurorelativity. The chronoarchitecture of the brain from the neuronal rather than the observer's perspective. Trends in Cognitive Sciences, 17(2), 51-52. http://doi.org/10.1016/j.tics.2012.12.005

Steglich, C., \& Neumann, O. (2000). Temporal, but not spatial, context modulates a masked prime's effect on temporal order judgment, but not on response latency. Psychological Research.

Sternberg, S., \& Knoll, R. L. (1973). The Perception of Temporal Order: Fundamental Issues and a General Model. In S. Kornblum (Ed.), Attention and performance IV (pp. 629685). New York: Academic Press.

Sweet, A. L. (1953). Temporal discrimination by the human eye. The American Journal of Psychology, 66(2), 185-198. http://doi.org/10.2307/1418725

Tappe, T., Niepel, M., \& Neumann, O. (1994). A dissociation between reaction time to sinusoidal gratings and temporal-order judgment. Perception, 23(3), 335-47. 
Wehrhahn, C., \& Rapf, D. (1992). ON- and OFF-pathways form separate neural substrates for motion perception: psychophysical evidence. The Journal of Neuroscience, 12(6), 2247-2250. http://doi.org/10.1523/JNEUROSCI.12-06-02247.1992

Westheimer, G., \& McKee, S. (1977). Perception of temporal order in adjacent visual stimuli. Vision Research, 17(8), 887-892.

Whitney, D., \& Murakami, I. (1998). Latency difference, not spatial extrapolation. Nature Neuroscience, 1(2), 656-657.

Whitney, D., Murakami, I., \& Cavanagh, P. (2000). Illusory spatial offset of a flash relative to a moving stimulus is caused by differential latencies for moving and flashed stimuli. Vision Research, 40(2), 137-49.

Wittmann, M., van Wassenhove, V., Craig, A. D., \& Paulus, M. P. (2010). The neural substrates of subjective time dilation. Frontiers in Human Neuroscience, 4(February), 1-9. http://doi.org/10.3389/neuro.09.002.2010

Yarrow, K., \& Arnold, D. H. (2015). The Timing of Experiences: How Far Can We Get with Simple Brain time Models? In B. Mölder, V. Arstila, \& P. Øhrstrøm (Eds.), Philosophy and Psychology of Time. Dordrecht, The Netherlands: Springer.

Yarrow, K., Jahn, N., Durant, S., \& Arnold, D. H. (2011). Shifts of criteria or neural timing? The assumptions underlying timing perception studies. Consciousness and Cognition, 20(4), 1518-1531. http://doi.org/10.1016/j.concog.2011.07.003

Zeki, S. (2007). A theory of micro-consciousness. The Blackwell Companion to Consciousness.

Zeki, S., \& Bartels, A. (1999). Toward a theory of visual consciousness. Consciousness and Cognition, 8(2), 225-59. 
${ }^{\mathrm{i}}$ It is worth noting, however, that the thesis is widely endorsed in science. For instance, Wolfgang Köhler (62 1947) already argued that "experienced order in time is always structurally identical with a functional order in the sequence of correlated brain processes." More recently a version of the thesis has figured in the explanations perceptual simultaneity (Kopinska \& Harris 2004), duration estimation and reproduction (Reutimann, Yakovlev, Fusi, \& Senn 2004; Wittmann, van Wassenhove, Craig, \& Paulus 2010), spatiotemporal illusions (Arstila 2015; Whitney \& Murakami 1998; Whitney, Murakami, \& Cavanagh 2000), and timing of visual feature changes (Arnold \& Wilcock 2007; Moutoussis \& Zeki 1997).

${ }^{\text {ii }}$ For the discussion of the thesis as such, see (e.g., Grush 2007; Kelly 2005; Kiverstein \& Arstila 2013; Mölder 2014). For the discussion of the thesis complemented with additional qualifications, see (e.g., Grush 2009; Lee 2014; Phillips 2014).

iii At the latter part of the paper, they also argue that if Benjamin Libet's results suggesting the delay of conscious intention and the backward referrals of time are sound, then the model of consciousness they advocate would explain them better than the Cartesian theater model of consciousness.

iv On this topic, it is interesting to note that even though many of the almost thirty commentators of Dennett and Kinsbourne's paper had reservations about the Multiple Drafts model, only one of them challenged the view that subjective time and brain time can come apart.

${ }^{\vee}$ Obviously, if one rejects, as many proponents of the brain-time view reject, the assumption that a temporal comparator tries to deduce the event time from the brain time, then this problem does not emerge.

${ }^{\text {vi }}$ See Holcombe 2015 for a thorough discussion of the evidence supporting this explanation in various unimodal and crossmodal temporal order tasks.

vii Since this position emphasizes the importance of simple latency differences, it has also been called as the simple brain-time view (Yarrow \& Arnold 2015). In the debates concerning spatiotemporal illusions, it is referred to as the latency difference hypothesis, the simple differential latency hypothesis, and the simple differential lag model (Arnold, Ong, \& Roseboom 2009; Chappell \& Hine 2004; Whitney \& Murakami 1998; Whitney et al. 2000). 